\title{
A Note on the Relative Ritt Order of Entire Functions Represented by Vector Valued Dirichlet Series
}

\author{
Sanjib Kumar Datta*, Tanmay Biswas and Pranab Das \\ (Communicated by Nihal YILMAZ ÖZGÜR)
}

\begin{abstract}
In this paper we investigate some growth properties of entire functions represented by a vector valued Dirichlet series on the basis of relative Ritt order and relative Ritt lower order.
\end{abstract}

Keywords: Vector valued Dirichlet series (VVDS); relative Ritt order; relative Ritt lower order; growth.

AMS Subject Classification (2010): Primary: 30B50; Secondary: 30D15; 30D99.

*Corresponding author

\section{Introduction, Definitions and Notations.}

Let $f(s)$ be an entire function of the complex variable $s=\sigma+i t$ ( $\sigma$ and $t$ are real variables) defined by everywhere absolutely convergent vector valued Dirichlet series

$$
f(s)=\sum_{n=1}^{\infty} a_{n} e^{s \lambda_{n}}
$$

where $a_{n}$ 's belong to a Banach space $(E,\|\|$.$) and \lambda_{n}$ 's are non-negative real numbers such that $0<\lambda_{n}<$ $\lambda_{n+1}(n \geq 1), \lambda_{n} \rightarrow \infty$ as $n \rightarrow \infty$ and satisfy the conditions

$$
\limsup _{n \rightarrow \infty} \frac{\log n}{\lambda_{n}}=D<\infty
$$

and

$$
\limsup _{n \rightarrow \infty} \frac{\log \left\|a_{n}\right\|}{\lambda_{n}}=-\infty .
$$

If $\sigma_{a}$ and $\sigma_{c}$ denote respectively the abscissa of convergence and absolute convergence of (1.1), then in this case clearly $\sigma_{a}=\sigma_{c}=\infty$.

The function $M_{f}(\sigma)$ known as maximum modulus function corresponding to an entire function $f(s)$ defined by (1.1) is written as follows:

$$
M_{f}(\sigma)=\underset{-\infty<t<\infty}{l . u . b .}|f(\sigma+i t)| .
$$

In the sequel the following two notations are used:

$$
\begin{aligned}
& \log ^{[k]} x=\log \left(\log ^{[k-1]} x\right) \text { for } k=1,2,3, \cdots ; \\
& \log ^{[0]} x=x
\end{aligned}
$$

Received : 03-August-2015, Accepted : 13-January-2016 
and

$$
\begin{aligned}
& \exp ^{[k]} x=\exp \left(\exp ^{[k-1]} x\right) \text { for } k=1,2,3, \cdots ; \\
& \exp ^{[0]} x=x
\end{aligned}
$$

Taking this into account, the Ritt order (See [1]), of $f(s)$, denoted by $\rho_{f}$, which is generally used in computational purpose, is defined in terms of the growth of $f(s)$ with respect to the exp $\exp z$ function as follows:

$$
\rho_{f}=\limsup _{\sigma \rightarrow \infty} \frac{\log \log M_{f}(\sigma)}{\log \log M_{\exp \exp z}(\sigma)}=\limsup _{\sigma \rightarrow \infty} \frac{\log ^{[2]} M_{f}(\sigma)}{\sigma} .
$$

Similarly, one can define the Ritt lower order of $f(s)$, denoted by $\lambda_{f}$ in the following manner:

$$
\lambda_{f}=\liminf _{\sigma \rightarrow \infty} \frac{\log \log M_{f}(\sigma)}{\log \log M_{\exp \exp z}(\sigma)}=\liminf _{\sigma \rightarrow \infty} \frac{\log ^{[2]} M_{f}(\sigma)}{\sigma} .
$$

Further an entire function $f(s)$ defined by (1.1) is said to be of regular Ritt growth if its Ritt order coincides with its Ritt lower order. Otherwise $f(s)$ is said to be of irregular Ritt-growth.

During the past decades, several authors \{e.g., cf., [1], [2], [3], [5], [7]\} have made intensive investigations on the properties of entire Dirichlet series related to Ritt order. Further, Srivastava [6] defined different growth parameters such as order and lower order of entire functions represented by vector valued Dirichlet series. He also obtained the results for coefficient characterization of order.

Srivastava [4] introduced the relative Ritt order between two entire functions represented by vector valued Dirichlet series to avoid comparing growth just with $\exp \exp z$ as follows:

$$
\begin{aligned}
\rho_{g}(f) & =\inf \left\{\mu>0: M_{f}(\sigma)<M_{g}(\sigma \mu) \text { for all } \sigma>\sigma_{0}(\mu)\right\} \\
& =\limsup _{\sigma \rightarrow \infty} \frac{M_{g}^{-1} M_{f}(\sigma)}{\sigma} .
\end{aligned}
$$

Similarly, one can define the relative Ritt lower order of $f(s)$ with respect to $g(s)$, denoted by $\lambda_{g}(f)$ in the following manner:

$$
\lambda_{g}(f)=\liminf _{\sigma \rightarrow \infty} \frac{M_{g}^{-1} M_{f}(\sigma)}{\sigma} .
$$

For entire functions, the notions of thier growth indicators such as Ritt order are classical in complex analysis and during the past decades, several researchers have already been exploring their studies in different directions using the classical growth indicators. But at that time, the concepts of relative Ritt order of entire functions and as well as their technical advantages of not comparing with the growths of exp exp $z$ are not at all known to the researchers of this area. Therefore the studies of the growths of composite entire functions in the light of their relative Ritt order are the prime concern of this paper. Actually in this paper we establish some newly developed results related to the growth rates of composite entire functions on the basis of their relative Ritt order (respectively relative Ritt lower order).

\section{Theorems.}

In this section we present the main results.

Theorem 2.1. Let $f$ and $g$ be any two entire functions represented by vector valued Dirichlet series such that $0<\lambda_{f} \leq \rho_{f}<$ $\infty$ and $0<\lambda_{g} \leq \rho_{g}<\infty$. Then

$$
\frac{\lambda_{f}}{\rho_{g}} \leq \lambda_{g}(f) \leq \min \left\{\frac{\lambda_{f}}{\lambda_{g}}, \frac{\rho_{f}}{\rho_{g}}\right\} \leq \max \left\{\frac{\lambda_{f}}{\lambda_{g}}, \frac{\rho_{f}}{\rho_{g}}\right\} \leq \rho_{g}(f) \leq \frac{\rho_{f}}{\lambda_{g}} .
$$

Proof. From the definitions of $\rho_{f}$ and $\lambda_{f}$, we have for all sufficiently large values of $\sigma$ that

$$
\begin{aligned}
& M_{f}(\sigma) \leq \exp ^{[2]}\left\{\left(\rho_{f}+\varepsilon\right) \sigma\right\} \\
& M_{f}(\sigma) \geq \exp ^{[2]}\left\{\left(\lambda_{f}-\varepsilon\right) \sigma\right\}
\end{aligned}
$$


and also for a sequence of values of $\sigma$ tending to infinity we get

$$
\begin{aligned}
& M_{f}(\sigma) \geq \exp ^{[2]}\left\{\left(\rho_{f}-\varepsilon\right) \sigma\right\}, \\
& M_{f}(\sigma) \leq \exp ^{[2]}\left\{\left(\lambda_{f}+\varepsilon\right) \sigma\right\} .
\end{aligned}
$$

Similarly from the definitions of $\rho_{g}$ and $\lambda_{f}$, it follows for all sufficiently large values of $\sigma$ that

$$
\begin{aligned}
M_{g}(\sigma) & \leq \exp ^{[2]}\left\{\left(\rho_{g}+\varepsilon\right) \sigma\right\} \\
i . e ., \sigma & \leq M_{g}^{-1}\left[\exp ^{[2]}\left\{\left(\rho_{g}+\varepsilon\right) \sigma\right\}\right] \\
\text { i.e., } M_{g}^{-1}(\sigma) & \geq\left[\frac{\log ^{[2]} \sigma}{\left(\rho_{g}+\varepsilon\right)}\right], \\
M_{g}(\sigma) & \geq \exp ^{[2]}\left\{\left(\lambda_{g}-\varepsilon\right) \sigma\right\} \\
\text { i.e., } M_{g}^{-1}(\sigma) & \leq\left[\frac{\log ^{[2]} \sigma}{\left(\lambda_{g}-\varepsilon\right)}\right]
\end{aligned}
$$

and for a sequence of values of $\sigma$ tending to infinity we obtain

$$
\begin{aligned}
M_{g}(\sigma) & \geq \exp ^{[2]}\left\{\left(\rho_{g}-\varepsilon\right) \sigma\right\} \\
\text { i.e. } M_{g}^{-1}(\sigma) & \leq\left[\frac{\log ^{[2]} \sigma}{\left(\rho_{g}-\varepsilon\right)}\right], \\
M_{g}(\sigma) & \leq \exp ^{[2]}\left\{\left(\lambda_{g}+\varepsilon\right) \sigma\right\} \\
\text { i.e., } M_{g}^{-1}(\sigma) & \geq\left[\frac{\log ^{[2]} \sigma}{\left(\lambda_{g}+\varepsilon\right)}\right] .
\end{aligned}
$$

Now from (2.3) and in view of (2.5), we get for a sequence of values of $\sigma$ tending to infinity we get

$$
\begin{gathered}
M_{g}^{-1} M_{f}(\sigma) \geq M_{g}^{-1}\left[\exp ^{[2]}\left\{\left(\rho_{f}-\varepsilon\right) \sigma\right\}\right] \\
\text { i.e., } M_{g}^{-1} M_{f}(\sigma) \geq\left[\frac{\log ^{[2]} \exp ^{[2]}\left\{\left(\rho_{f}-\varepsilon\right) \sigma\right\}}{\left(\rho_{g}+\varepsilon\right)}\right] \\
=\frac{\left(\rho_{f}-\varepsilon\right)}{\left(\rho_{g}+\varepsilon\right)} \sigma \\
\text { i.e., } \frac{M_{g}^{-1} M_{f}(r)}{\sigma} \geq \frac{\left(\rho_{f}-\varepsilon\right)}{\left(\rho_{g}+\varepsilon\right)} .
\end{gathered}
$$

As $\varepsilon>0$ is arbitrary, it follows that

$$
\rho_{g}(f) \geq \frac{\rho_{f}}{\rho_{g}} .
$$

Analogously from (2.2) and in view of (2.8), it follows for a sequence of values of $\sigma$ tending to infinity that

$$
\begin{aligned}
M_{g}^{-1} M_{f}(\sigma) \geq & M_{g}^{-1}\left[\exp ^{[2]}\left\{\left(\lambda_{f}-\varepsilon\right) \sigma\right\}\right] \\
\text { i.e., } M_{g}^{-1} M_{f}(\sigma) & \geq\left[\frac{\log ^{[2]} \exp ^{[2]}\left\{\left(\lambda_{f}-\varepsilon\right) \sigma\right\}}{\left(\lambda_{g}+\varepsilon\right)}\right] \\
& =\frac{\left(\lambda_{f}-\varepsilon\right)}{\left(\lambda_{g}+\varepsilon\right)} \sigma
\end{aligned}
$$




$$
\text { i.e., } \frac{M_{g}^{-1} M_{f}(r)}{\sigma} \geq \frac{\left(\lambda_{f}-\varepsilon\right)}{\left(\lambda_{g}+\varepsilon\right)} .
$$

Since $\varepsilon(>0)$ is arbitrary, we get from above that

$$
\rho_{g}(f) \geq \frac{\lambda_{f}}{\lambda_{g}}
$$

Again in view of (2.6), we have from (2.1) for all sufficiently large values of $\sigma$ that

$$
\begin{gathered}
M_{g}^{-1} M_{f}(\sigma) \leq M_{g}^{-1}\left[\exp ^{[2]}\left\{\left(\rho_{f}+\varepsilon\right) \sigma\right\}\right] \\
\text { i.e., } M_{g}^{-1} M_{f}(\sigma) \leq\left[\frac{\log ^{[2]} \exp ^{[2]}\left\{\left(\rho_{f}+\varepsilon\right) \sigma\right\}}{\left(\lambda_{g}-\varepsilon\right)}\right] \\
=\frac{\left(\rho_{f}+\varepsilon\right)}{\left(\lambda_{g}-\varepsilon\right)} \sigma \\
\text { i.e., } \frac{M_{g}^{-1} M_{f}(\sigma)}{\sigma} \leq \frac{\left(\rho_{f}+\varepsilon\right)}{\left(\lambda_{g}-\varepsilon\right)} .
\end{gathered}
$$

Since $\varepsilon(>0)$ is arbitrary, we obtain that

$$
\rho_{g}(f) \leq \frac{\rho_{f}}{\lambda_{g}}
$$

Again from (2.2) and in view of (2.5) with the same reasoning we get that

$$
\lambda_{g}(f) \geq \frac{\lambda_{f}}{\rho_{g}} .
$$

Also in view of (2.7), we get from (2.1) for a sequence of values of $\sigma$ tending to infinity that

$$
\begin{gathered}
M_{g}^{-1} M_{f}(\sigma) \leq M_{g}^{-1}\left[\exp ^{[2]}\left\{\left(\rho_{f}+\varepsilon\right) \sigma\right\}\right] \\
\text { i.e., } M_{g}^{-1} M_{f}(\sigma) \leq\left[\frac{\log ^{[2]} \exp ^{[2]}\left\{\left(\rho_{f}+\varepsilon\right) \sigma\right\}}{\left(\rho_{g}-\varepsilon\right)}\right] \\
=\frac{\left(\rho_{f}+\varepsilon\right)}{\left(\rho_{g}-\varepsilon\right)} \sigma \\
\text { i.e., } \frac{M_{g}^{-1} M_{f}(\sigma)}{\sigma} \leq \frac{\left(\rho_{f}+\varepsilon\right)}{\left(\rho_{g}-\varepsilon\right)} .
\end{gathered}
$$

Since $\varepsilon(>0)$ is arbitrary, we get from above that

$$
\lambda_{g}(f) \leq \frac{\rho_{f}}{\rho_{g}}
$$

Similarly from (2.4) and in view of (2.6), it follows for a sequence of values of $\sigma$ tending to infinity that

$$
\begin{aligned}
M_{g}^{-1} M_{f}(\sigma) \leq & M_{g}^{-1}\left[\exp ^{[2]}\left\{\left(\lambda_{f}+\varepsilon\right) \sigma\right\}\right] \\
\text { i.e., } M_{g}^{-1} M_{f}(\sigma) & \leq\left[\frac{\log ^{[2]} \exp ^{[2]}\left\{\left(\lambda_{f}+\varepsilon\right) \sigma\right\}}{\left(\lambda_{g}-\varepsilon\right)}\right] \\
& =\frac{\left(\lambda_{f}+\varepsilon\right)}{\left(\lambda_{g}-\varepsilon\right)} \sigma
\end{aligned}
$$




$$
\text { i.e., } \frac{M_{g}^{-1} M_{f}(\sigma)}{\sigma} \leq \frac{\left(\lambda_{f}+\varepsilon\right)}{\left(\lambda_{g}-\varepsilon\right)}
$$

As $\varepsilon(>0)$ is arbitrary, we obtain from above that

$$
\lambda_{g}(f) \leq \frac{\lambda_{f}}{\lambda_{g}} .
$$

The theorem follows from $(2.9),(2.10),(2.11),(2.12),(2.13)$ and (2.14).

Corollary 2.1. Let $f$ be an entire function represented by vector valued Dirichlet series with Ritt order $\rho_{f}$ and Ritt lower order $\lambda_{f}$. Also let $g$ be an entire function of regular Ritt growth. Then

$$
\lambda_{g}(f)=\frac{\lambda_{f}}{\rho_{g}} \text { and } \rho_{g}(f)=\frac{\rho_{f}}{\rho_{g}} .
$$

In addition, if $\rho_{f}=\rho_{g}$, then

$$
\rho_{g}(f)=\lambda_{f}(g)=1 .
$$

Corollary 2.2. Let $f$ and $g$ be any two entire functions represented by vector valued Dirichlet series with regular Ritt growth. Then

$$
\lambda_{g}(f)=\rho_{g}(f)=\frac{\rho_{f}}{\rho_{g}} .
$$

Corollary 2.3. Let $f$ and $g$ be any two entire functions represented by vector valued Dirichlet series with regular Ritt growth. Also suppose that $\rho_{f}=\rho_{g}$. Then

$$
\lambda_{g}(f)=\rho_{g}(f)=\lambda_{f}(g)=\rho_{f}(g)=1 .
$$

Corollary 2.4. Let $f$ and $g$ be any two entire functions represented by vector valued Dirichlet series with regular Ritt growth and $0<\lambda_{g}<\rho_{g}<\infty$ respectively. Then

$$
\rho_{g}(f) \cdot \rho_{f}(g)=\lambda_{g}(f) \cdot \lambda_{f}(g)=1 .
$$

Corollary 2.5. Let $f$ and $g$ be any two entire functions represented by vector valued Dirichlet series and either $f$ is not of regular Ritt growth or $g$ is not of regular Ritt growth, then

$$
\lambda_{g}(f) \cdot \lambda_{f}(g)<1<\rho_{g}(f) \cdot \rho_{f}(g) .
$$

Corollary 2.6. Let $f$ be an entire function represented by vector valued Dirichlet series with $0<\lambda_{f}<\rho_{f}<\infty$. Then for any entire function $g$ represented by vector valued Dirichlet series,

$$
\begin{aligned}
\text { (i) } \lambda_{g}(f) & =\infty \text { when } \rho_{g}=0 \\
\text { (ii) } \rho_{g}(f) & =\infty \text { when } \lambda_{g}=0 \\
\text { (iii) } \lambda_{g}(f) & =0 \text { when } \rho_{g}=\infty
\end{aligned}
$$

and

$$
\text { (iv) } \rho_{g}(f)=0 \text { when } \lambda_{g}=\infty .
$$

Corollary 2.7. Let $g$ be an entire function represented by vector valued Dirichlet series with $0<\lambda_{g}<\rho_{g}<\infty$. Then for any entire function $f$ represented by vector valued Dirichlet series,

$$
\begin{aligned}
\text { (i) } \rho_{g}(f) & =0 \text { when } \rho_{f}=0 \\
\text { (ii) } \lambda_{g}(f) & =0 \text { when } \lambda_{f}=0 \\
\text { (iii) } \rho_{g}(f) & =\infty \text { when } \rho_{f}=\infty
\end{aligned}
$$

and

(iv) $\lambda_{g}(f)=\infty$ when $\lambda_{f}=\infty$.

\section{Acknowledgment}

The authors are thankful to the referees for their valuable suggestions towards the improvement of the paper. 


\section{References}

[1] Rahman, Q. I., The Ritt order of the derivative of an entire function. Annales Polonici Mathematici 17 (1965), 137-140.

[2] Rajagopal, C. T. and Reddy, A. R., A note on entire functions represented by Dirichlet series. Annales Polonici Mathematici 17 (1965), 199-208.

[3] Ritt, J. F., On certain points in the theory of Dirichlet series. Amer. Jour. Math. 50 (1928), 73-86.

[4] Srivastava, G. S., A note on relative type of entire functions represented by vector valued dirichlet series. Journal of Classicial Analysis 2 (2013), no. 1, 61-72.

[5] Srivastava, G. S. and Sharma, A., On generalized order and generalized type of vector valued Dirichlet series of slow growth. Int. J. Math. Archive 2 (2011), no. 12, 2652-2659.

[6] Srivastava, B. L., A study of spaces of certain classes of vector valued Dirichlet series. Thesis, I. I. T., Kanpur, 1983.

[7] Srivastav, R. P. and Ghosh, R. K., On entire functions represented by Dirichlet series. Annales Polonici Mathematici 13 (1963), 93-100.

\section{Affiliations}

SANJIB KUMAR DATTA

ADDRESS: University of Kalyani, Dept. of Mathematics, PIN- 741235, West Bengal-India.

E-MAIL: sanjib_kr_datta@yahoo.co.in

TANMAY BISWAS

AdDress: PIN- 741101, West Bengal-India.

E-MAIL: tanmaybiswas_math@rediffmail.com

PRANAB DAS

ADDRESS: University of Kalyani, Dept. of Mathematics, PIN- 741235, West Bengal-India.

E-MAIL: pranabdas90@gmail.com 